\title{
Entornos virtuales de aprendizaje adoptados en la universidad ante el COVID-19
}

\section{Virtual learning environments adopted by the university due to COVID-19}

DOI: https://doi.org/10.32870/dse.v0i22.829

Ulises Delgado Sánchez*

Fernanda Gabriela Martínez Flores**

\begin{abstract}
Resumen
La educación en tiempos de crisis se ha presentado por diversas causas, entre las cuales destaca la sanitaria. Actualmente, el mundo vive una pandemia por coronavirus que ha llevado a las instituciones educativas a suspender las clases presenciales en la mayor parte del mundo. En este contexto, nuestro objetivo fue identificar los entornos virtuales de aprendizaje (EVA) a los que han recurrido profesores y alumnos de educación superior ante esta situación, revelando las correlaciones entre estos y las prácticas educativas. Participaron 425 estudiantes de diversas universidades, se les aplicó el Cuestionario de Prácticas Educativas Virtuales por COVID-19; encontrándose principalmente que los docentes recurrieron al uso de plataformas virtuales institucionales, así como la mensajería móvil instantánea y medios asíncronos de comunicación personal complementarios. Se encontró que el apoyo de los EVA institucionales está correlacionado con la retroalimentación a los alumnos, por lo que pueden considerarse EVA eficaces. Estos resultados permiten reconocer que han sobresalido algunos EVA formales y otros informales o de uso personal, que en menor medida también han dado soporte a las tareas educativas.
\end{abstract}

Palabras clave: crisis educativa - (por) enfermedades - EVA - técnicas de medición - estudiantes - educación superior.

\begin{abstract}
Education in times of crisis is due to various causes, among them the sanitary. The world is currently experiencing a coronavirus pandemic that has led educational institutions to suspend face-to-face classes in most of the world. In this context, our aim was to identify the virtual learning environments (VLE) that teachers and students of higher education have resorted to, revealing the correlations between these environments and educational practices. The Virtual Educational Practices Questionnaire for COVID-19 was
\end{abstract}

\footnotetext{
* Doctor en Psicología. Profesor-investigador de tiempo completo. Centro de Investigación Transdisciplinar en Psicología. Universidad Autónoma del Estado de Morelos. México. ulises.delgado@uaem.mx

** Doctora en Psicología. Académica de la Facultad de Comunicación Humana. Universidad Autónoma del Estado de Morelos. México. maga. mtzf@gmail.com
} 
applied to 425 students from various universities, and the main finding was that the teachers resorted to the use of institutional virtual platforms, as well as instant mobile messaging and complementary asynchronous means of personal communication. Support from institutional VAS correlated with feedback to students, so they can be considered effective. These results allow us to recognize that some formal VAS have excelled, and other informal or personal means of communication have had a lesser impact on educational task support.

Key words: educational crisis - (due to) diseases - EVA - measurement techniques - students - higher education.

\section{Introducción}

La pandemia por coronavirus (COVID-19) que ha generado una crisis sanitaria está repercutiendo en diferentes ámbitos de la sociedad; la educación no queda exenta de las consecuencias de este problema sanitario al verse afectados 1,600 millones de alumnos en más de 190 países en todos los continentes (ONU, 2020). En el mundo se han suspendido clases en todos los niveles educativos, por lo que las escuelas, los docentes, los estudiantes y el personal administrativo están enfrentando el reto de continuar el proceso formativo desde el hogar de cada estudiante, con apoyo de los recursos digitales. Pero, ¿cuáles son esos recursos digitales que se están implementando? ¿tienen las universidades posibilidades de continuar con su proceso formativo, y pasar de las clases presenciales a la virtuales de manera eficiente? Estos son algunos de los retos a los que se enfrenta la educación en este nuevo milenio, afectado por la incertidumbre de una contingencia sanitaria y sus consecuencias en los contextos educativos.

Lo anterior nos hace mirar hacia la educación en situaciones de crisis. La UNESCO (2020) anticipaba el aumento de los conflictos armados, los desastres naturales y las epidemias en distintas partes del planeta, todos ellos vulnerando y en algunos casos impidiendo la continuidad educativa de millones de niños, adolescentes y adultos. Álvarez et al. (2020) describen la implementación de medios de primera generación para continuar con los procesos de enseñanzaaprendizaje en esta pandemia, entre ellos están los materiales impresos, la radio y televisión, así como medios de segunda generación como son las plataformas y sistemas de gestión de aprendizaje, destacando que en México se aplican principalmente los contenidos digitales, la radio o la televisión. En este contexto, cobra especial importancia la sugerencia de Quirós (2009): promover a gran escala el uso de los recursos didácticos digitales por profesores y estudiantes, para favorecer el aprendizaje colaborativo en dicho entorno. Los entornos virtuales de aprendizaje (EVA), entre los que se encuentra el uso de plataformas e-learning, comprenden una amplia variedad de recursos, como la plataforma Moodle, Flipped Classroom, Google Classroom, Edmodo, Blackboard-collaborate, así como diversos recursos disponibles en la Web, que fueron concebidos solo con un fin comunicacional.

DiáloQos año 12 | número 22 | enero-junio 2021 | ISSN 2007-2171 
En relación con el empleo de la plataforma Moodle, Del Prete y Cabrero (2019) encontraron que el uso que le dan los profesores universitarios es de repositorio de objetos de aprendizaje o de recolección de diferente información de los estudiantes. Mientras que Mejía, Hidalgo, Rosero y Yugsan (2018) concluyen que la plataforma Edmodo se ha utilizado como complementaria a las asignaturas presenciales, al señalar que en una clase de inglés los estudiantes del grupo experimental que utilizaron esta plataforma presentaron mejores resultados en el aprendizaje y precisión de contenidos que los del grupo control. El uso de Google Classroom, como demostró Lorenzo (2019), es otra herramienta en la educación media superior, la cual se ha usado paralelamente con Google Drive, Canva, Savia Digital, Blender, YouTube, entre otros; destaca que 79\% de los estudiantes de dicha investigación utilizaron esta plataforma para aprender un determinado contenido en la materia de inglés.

Otro EVA es el Flipped Classroom, el cual, como subrayan Aguilera, Manzano, Martínez, Lozano y Casiano (2017), permite la clase invertida y es un excelente soporte en cualquier contexto, haciendo cada vez más cercano el ambiente de una clase presencial, pero en un entorno virtual. En los hallazgos de Ferreriro, Garambullo y Brito (2013) la plataforma Blackboard ha resultado idónea como apoyo en el flujo de información entre los estudiantes y el docente, permitiendo la administración del tiempo y un adecuado uso de los recursos por parte de ambos. Por otra parte, el Microsoft Teams es un recurso gratuito y permite un espacio de comunicación, con videollamadas, videoconferencias, así como tutorías virtuales y clases invertidas (Microsoft, 2020).

Un estudio de Díaz (2009) destaca que las plataformas institucionales son plataformas de desarrollo propio, no son de acceso al público en general, por lo que solo responden a necesidades y situaciones educativas concretas, lo que hace poco atractivos los estudios sobre ellas. A estas afirmaciones se suma la de Ardila (2011), quien concluye que los actores del uso de las plataformas institucionales tienen como objetivo la producción, administración de datos, información y conocimiento, que permiten intercambiar y compartir conocimientos y la construcción de los mismos en diferentes lugares y tiempos.

De acuerdo con Ricardo et al. (2017), el Zoom entrará dentro de los EVA, ya que su uso permite difundir videoconferencias de contenido académico, cultural, artístico, tecnológico y de innovación; por lo que es ampliamente usado en universidades colombianas que lo han incluido como herramienta de documentales y materiales que pueden utilizar los docentes; y además es vinculable con el canal de YouTube, Twitter o Facebook. También hay reportes de que el Zoom ha sido una opción didáctica en universidades de Argentina (Dallera, Tenaglía, 2000) que se ha constituido en un complemento para el estudio y destacan su utilidad como herramienta didáctica en la enseñanza que permite mejorar los conocimientos de los estudiantes.

El uso de teléfonos celulares se puede señalar como una herramienta digital que ha permitido la implementación de los EVA al alcance de un gran número de personas que no dispo- 
nen de computadoras de escritorio o portátiles. En un estudio reciente, realizado en Chile por Aranciba, Cabrero y Valdivia (2019), se identificó que $77.9 \%$ de los estudiantes y $74.1 \%$ de los docentes consideran que los celulares son una herramienta para la clase que permite buscar información relevante en internet, así como $64.4 \%$ de los estudiantes y $49.2 \%$ de los docentes están de acuerdo en que por medio de estos se puede acceder a recursos digitales; sin embargo, $93 \%$ de los estudiantes consideran que el celular no es una herramienta efectiva de aprendizaje y $88.9 \%$ de los docentes coinciden con ello. Es importante destacar que los reportes hasta el momento, aunque contradictorios, no ponen en duda la importancia de esta herramienta tecnológica para fines de comunicación, a través de una variedad de redes sociales como YouTube, WhatsApp, correo electrónico, entre otros.

No puede dejar de considerarse el caso de Facebook, que se diseñó como un espacio personal, red social y sin un perfil académico; sin embargo, Abúndez, Fernández, Meza y Álamo (2015) encontraron que 39\% de los estudiantes de nivel medio superior consideran necesario incorporar esta aplicación para tareas escolares, identificándose que $46.4 \%$ de los estudiantes están de acuerdo en que este medio agiliza la retroalimentación por parte del docente. Blanco y López (2017) utilizaron la red social de Instagram para trabajar la temática de poesías en el Centro de Educación para Personas Adultas en Madrid, y encontraron que la posibilidad de uso de material visual de esta red permite que los estudiantes entiendan mejor y tengan retroalimentación.

Respecto a las redes sociales digitales, el sitio web YouTube se ha utilizado como un apoyo para la educación. Se han realizado estudios donde se emplea como herramienta para impartir piezas de contenido de unidades de aprendizaje con estudiantes de nivel medio superior, siendo mayor su eficiencia cuando es implementado de manera complementaria a los cursos regulares, lo cual favorece una mejora en el promedio obtenido por los alumnos (Pérez, Cuecuecha, 2019; Rodríguez, Fernández, 2017). Aparentemente, es posible que se incluya el uso de YouTube como un recurso de contenido para apoyar aprendizaje en línea.

Una aplicación prácticamente presente en todo teléfono móvil es el WhatsApp, sin embargo, no ha sido incluida en forma relevante como un ambiente educativo; esta aplicación, como han señalado Fietyat, Ahmad y Yudi (2017), es muy versátil ya que puede utilizarse por medio del Whatsapp Web en computadores de escritorio, haciéndola una aplicación multiplataforma. Suárez (2018) afirma que una de las ventajas de su uso en la educación radica en la posibilidad de dar anuncios sobre cuestiones educativas, disipar dudas, permitir la retroalimentación de una manera rápida, así como tener accesibilidad de materiales formativos en diversos formatos; fue Trejo (2018) quien encontró que estudiantes universitarios consideran que por medio de este recurso es más efectiva la comunicación con sus profesores, por lo que el uso de esta aplicación permite una comunicación eficiente para la enseñanza.

Por otra parte, Puerta y Sánchez (2010) plantean que el correo electrónico permite crear un proceso comunicativo presentando propósitos definidos y muy claros por parte del destinata-

Diálo@os

sobre Educación año 12 | número 22 | enero-junio 2021 | ISSN 2007-2171 
rio, por lo que este medio puede tener funciones informativas, didácticas y comunicativas; aún con ello, se ha encontrado que los docentes no dan respuesta a los correos con rapidez, dejan a los estudiantes en desorientación e incertidumbre. Luis (2014) encontró en un estudio que $25 \%$ de los profesores utilizan poco el correo para comunicarse con sus alumnos, $40 \%$ lo utilizan mucho, mientras que $35 \%$ de los docentes lo utilizan bastante.

Otros recursos que están en la web son los Blogs. Ruíz y Abella (2011) identificaron que la valoración que hacen estudiantes de educación superior sobre la utilidad educativa de estos es buena, $81 \%$ de los estudiantes presentaron una apreciación favorable para este recurso. De igual manera, de acuerdo con Ricardo et al. (2017), el Khan Academy se ha convertido en un sistema interactivo completo en la educación.

Es importante destacar que para autores como Del Prete, Cabrero y Halal (2018), la implementación de los EVA como herramientas tienen limitaciones derivadas del desconocimiento de su gestión e implementación por parte de los docentes, así como de las políticas institucionales para el fomento y ayuda en tales herramientas.

Ante la actual emergencia derivada de la pandemia por COVID-19, las instituciones educativas en todo el mundo se vieron en la necesidad de encontrar alternativas para impartir las clases presenciales. En el caso de los bachilleratos y las universidades públicas, nacionales y estatales, así como algunas universidades del sector privado, a pesar de contar con la infraestructura tecnológica para migrar los cursos presenciales a plataformas virtuales, no todas han avanzado de manera homogénea en una cultura digital, tanto de sus profesores como estudiantes. El reto de continuar con las actividades formativas ha requerido una respuesta súbita, pero eficaz. ¿Cuáles pueden ser los recursos comunicativos y educativos que se han elegido con mayor aceptación por las comunidades educativas en nuestro país y en algunos países latinoamericanos? Los objetivos de la presente exploración son: 1) identificar los recursos tecnológicos que se están implementando ante la contingencia del COVID-19 en Latinoamérica; 2) correlacionar los protocolos virtuales identificados, las indicaciones del profesor, la capacitación a los estudiantes y la retroalimentación recibida con los recursos tecnológicos implementados por la contingencia del COVID-19, y 3) identificar la correlación entre la implementación de los diferentes recursos tecnológicos.

\section{Método \\ Diseño de investigación \\ Transversal.}

Tipo de estudio

Exploratorio, descriptivo, correlacional. 


\section{Participantes}

Participaron 425 estudiantes entre 16 y 66 años, con una edad promedio de 22.21 años; de los cuales: 11 cuentan con educación media superior o carrera técnica, 363 de licenciatura, 5 de especialidad, 36 de maestría y 10 de doctorado. Respecto a su nacionalidad, 411 eran mexicanos, 3 colombianos, 1 costarricense, 3 estadunidenses, 2 cubanos, 1 ruso, 1 panameño, y 3 venezolanos. En total participaron 322 mujeres y 103 hombres.

\section{Instrumentos}

El Cuestionario de Prácticas Educativas Virtuales por COVID-19, consta de una sección de información sociodemográfica, una sección de 23 reactivos dicotómicos sobre las opciones adoptadas para llevar a cabo trabajo virtual y a distancia (EVA), y una sección sobre prácticas de las instituciones y los docentes ante las restricciones impuestas por el COVID-19.

\section{Procedimiento}

Se creó el cuestionario con el recurso tecnológico de Google Formularios, explicitando en la primera sección los objetivos del estudio, así como el formato de consentimiento informado. El cuestionario se difundió por medio de las redes sociales de Facebook y WhatsApp, a estudiantes de varios bachilleratos, licenciaturas y posgrados en México, con la solicitud de compartirlo con todos sus contactos que también fueran estudiantes actualmente. La recolección de datos comenzó el 26 de marzo y concluyó el 5 de abril del año en curso; se descargó la base de datos en el programa Excel 2016 y posteriormente, se trasladaron los datos al SPSS versión 23 para ser analizados, así como también se implementó el paquete estadístico G*Power. 3.1.9.7.

\section{Análisis de datos}

Los datos se obtuvieron de las frecuencias y porcentajes de los recursos virtuales reportados como empleados, y a partir de ello se realizó un análisis factorial para determinar la agrupación por factores para obtener el KMO y validez de contenido; posteriormente, se obtuvieron las correlaciones de Pearson para determinarlas, y los coeficientes de significancia. Por medio del G*Power se analizó el tamaño del efecto y la potencia estadística entre los EVA, y de estos, con la retroalimentación, capacitación e implementación de un protocolo virtual de clases.

\section{Resultados}

Se obtuvo que $98.6 \%$ (419) de los estudiantes reportaron estar en suspensión de clases, mientras que 1.4\% (6) indicaron estar en clases presenciales. A 96\% (408) de los estudiantes se les notificó formalmente el comienzo de un protocolo virtual de clases, mientras que 4\% (17) reportó no haber sido contactado para recibir información. De los que fueron notificados del cambio a al-

Diólopos sobre Educación

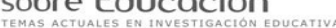

año 12 | número 22 | enero-junio 2021 | ISSN 2007-2171 
ternativas de aprendizaje virtual, 39.3 \% (167) indicó que se les brindó alguna capacitación para la modalidad virtual, mientras que $60.7 \%$ (258) no recibieron capacitación para llevar a cabo el seguimiento en modalidad virtual.

En la tabla 1 se pueden observar los diferentes EVA que se implementaron por parte de los docentes, así como la frecuencia y porcentaje de uso de los mismos.

Tabla 1. Uso de recursos tecnológicos en estudiantes ante la contingencia del COVID-19

\begin{tabular}{lllll}
\hline \multicolumn{1}{c}{ Recursos tecnológicos implementados } & \multicolumn{3}{c}{ Sí } & \multicolumn{3}{c}{ NO } \\
\hline & Frecuencia & $\%$ & Frecuencia & $\%$ \\
\cline { 2 - 5 } Plataforma virtual institucional & 308 & 72.5 & 117 & 27.5 \\
WhatsApp & 247 & 58.1 & 178 & 41.9 \\
Correo electrónico & 246 & 57.9 & 179 & 42.1 \\
Tareas en casa & 195 & 45.9 & 230 & 54.1 \\
Videollamadas & 155 & 36.5 & 270 & 63.5 \\
Facebook & 78 & 18.4 & 347 & 81.6 \\
Flipped Classroom & 75 & 17.6 & 350 & 82.4 \\
Moodle & 73 & 17.2 & 352 & 82.8 \\
Zoom & 56 & 13.2 & 369 & 86.8 \\
Videos educativos en YouTube realizados por el & 48 & 11.3 & 377 & 88.7 \\
docente & & & & \\
Google Classroom & 36 & 8.5 & 389 & 91.5 \\
Skype & 26 & 6.1 & 399 & 93.9 \\
Edmodo & 19 & 4.5 & 406 & 95.5 \\
Drive & 6 & 1.4 & 419 & 98.6 \\
Otro no mencionado en la lista & 6 & 1.4 & 419 & 98.6 \\
Blackboard & 1 & 0.7 & 422 & 99.3 \\
Microsoft Teams & 1 & 0.5 & 423 & 99.5 \\
Hangout Meet & 3 & 0.5 & 423 & 99.5 \\
Instagram & 2 & 0.2 & 424 & 99.8 \\
Clsco & 2 & 0.2 & 423 & 99.5 \\
Khan Academy & 1 & 0.2 & 424 & 99.8 \\
Blogger & & 424 & 99.8 \\
Programas de TV & & 0.2 & 100 \\
\hline & & 425 & \\
\hline
\end{tabular}

Con los datos recolectados se aplicó un análisis factorial exploratorio para determinar la agrupación por factores de los EVA, que resultaron ser tres, con una media de KMO de .57 y un total de la varianza explicada de 31.63. Se consideró como condición mínima la comunalidad 
de 0.30 , teniendo tres items como número de variables por factor y una muestra mínima de 400 casos, como indican Conway y Huffcutt (2003, en Lloret, Ferreres, Hernández, Tomás, 2014).

Tabla 2. Factores de los entornos virtuales de aprendizaje

\begin{tabular}{llll}
\hline Recursos tecnológicos implementados & Factor 1 & Factor 2 & Factor 3 \\
\hline Correo electrónico & .740 & & \\
WhatsApp & .685 & & \\
Facebook & .514 & & \\
Video llamadas & & .646 & \\
Videos YouTube & .641 & \\
Tareas en casa & .475 & \\
Zoom & .464 & \\
Plataformas institucionales & & .677 \\
Flipped Classroom & & .364 \\
Edmodo & & & .317 \\
\hline
\end{tabular}

En la tabla 3 se presentan las correlaciones ( $r$ ), los coeficientes de significancia estadística (Sig), tamaño del efecto ( $p$ ) y potencia estadística (1- $\beta$ ) de los resultados, en relación con la implementación de un protocolo virtual de clases, las indicaciones del profesor, la capacitación dada a los estudiantes, la retroalimentación con los EVA identificados en el análisis factorial exploratorio.

Tabla 3. Correlación de los entornos virtuales de aprendizaje con la implementación de un protocolo virtual de clases, capacitación, retroalimentación e indicaciones por los profesores

\begin{tabular}{|c|c|c|c|c|c|c|c|c|c|c|c|}
\hline & & $\begin{array}{c}\text { Whats- } \\
\text { App }\end{array}$ & $\begin{array}{l}\text { Face- } \\
\text { book }\end{array}$ & $\begin{array}{c}\text { Platafor- } \\
\text { mas } \\
\text { institucio- } \\
\text { nales }\end{array}$ & $\begin{array}{c}\text { Correo } \\
\text { elec- } \\
\text { trónico }\end{array}$ & $\begin{array}{c}\text { Video } \\
\text { Ilama- } \\
\text { das }\end{array}$ & $\begin{array}{l}\text { Ed- } \\
\text { modo }\end{array}$ & $\begin{array}{c}\text { Flipped } \\
\text { Clas- } \\
\text { sroom }\end{array}$ & $\begin{array}{c}\text { Tareas } \\
\text { en } \\
\text { casa }\end{array}$ & $\begin{array}{l}\text { Video } \\
\text { You- } \\
\text { Tube }\end{array}$ & Zoom \\
\hline \multirow{4}{*}{$\begin{array}{l}\text { Implemen- } \\
\text { tación de un } \\
\text { protocolo } \\
\text { virtual de } \\
\text { clases }\end{array}$} & $r$ & .021 & -.027 & .143 & .069 & .055 & -.072 & .000 & .019 & .035 & .009 \\
\hline & Sig & .66 & .575 & .003 & .155 & .259 & .138 & 1 & .692 & .473 & .861 \\
\hline & $\mathrm{p}$ & .14 & .16 & .37 & .26 & .23 & .26 & - & .13 & .18 & .09 \\
\hline & $1-\beta$ & .99 & .99 & .99 & .99 & .99 & .99 & - & .99 & .99 & .99 \\
\hline \multirow[t]{4}{*}{ Capacitación } & r & .078 & .042 & .205 & .159 & .071 & -.034 & .019 & .023 & .078 & .099 \\
\hline & Sig & .11 & .391 & .001 & .001 & .144 & .482 & 691 & .637 & .107 & .04 \\
\hline & $\mathrm{p}$ & .27 & .20 & .45 & .39 & .26 & .18 & .13 & .15 & .27 & $\cdot 31$ \\
\hline & $1-\beta$ & .99 & .99 & 1 & 1 & .99 & .99 & .99 & .99 & .99 & .99 \\
\hline
\end{tabular}

Diálǫos sobre Educación año 12 | número 22 | enero-junio 2021 | ISSN 2007-2171 
Entornos virtuales de aprendizaje adoptados en la universidad ante el COVID-19

\begin{tabular}{|c|c|c|c|c|c|c|c|c|c|c|c|}
\hline \multirow{4}{*}{$\begin{array}{l}\text { Retroalimen- } \\
\text { tación }\end{array}$} & $r$ & -.037 & .015 & .183 & .075 & .089. & .094 & .024 & -.095 & -.023 & 110 \\
\hline & Sig & .45 & $.75^{8}$ & .001 & .123 & .067 & .052 & .618 & .05 & .63 & .023 \\
\hline & $\mathrm{p}$ & .19 & .12 & .42 & .27 & .29 & .30 & .15 & .30 & .15 & .33 \\
\hline & $1-\beta$ & .99 & .98 & 1 & .99 & .99 & .99 & .99 & .99 & .99 & 99 \\
\hline \multirow{4}{*}{$\begin{array}{l}\text { Indicaciones } \\
\text { del profesor }\end{array}$} & $r$ & .016 & -.023 & .106 & .125 & -.007 & .039 & -.026 & .081 & .020 & -.011 \\
\hline & Sig & .744 & .641 & .030 & .010 & .885 & .429 & .588 & .096 & .676 & .814 \\
\hline & $p$ & .12 & .15 & .35 & .35 & .08 & .19 & .16 & .28 & .14 & .10 \\
\hline & $1-\beta$ & .89 & .89 & .92 & .91 & .92 & .91 & .89 & .92 & .89 & gو.8 \\
\hline
\end{tabular}

Se obtuvieron correlaciones significativas entre la implementación de un protocolo virtual de clases y el uso de plataformas institucionales; mientras que la capacitación resultó correlacionada con las plataformas institucionales, el correo electrónico y el uso de Zoom; la retroalimentación se correlacionó con plataformas institucionales y el Zoom, así como las indicaciones dadas por el profesor se correlacionaron con plataformas institucionales y el correo electrónico.

En la tabla 4 se presentan las correlaciones $(r)$, los coeficientes de significancia estadística $(S i g)$, tamaño del efecto $(p)$ y potencia estadística (1- $\beta$ ) entre los EVA identificados en el análisis factorial exploratorio.

Tabla 4. Correlaciones entre los entornos virtuales de aprendizaje implementados

\begin{tabular}{|c|c|c|c|c|c|c|c|c|c|c|c|}
\hline & & $\begin{array}{l}\text { Whats- } \\
\text { App }\end{array}$ & $\begin{array}{l}\text { Face- } \\
\text { book }\end{array}$ & $\begin{array}{c}\text { Platafor- } \\
\text { mas } \\
\text { institucio- } \\
\text { nales }\end{array}$ & $\begin{array}{l}\text { Correo } \\
\text { elec- } \\
\text { trónico }\end{array}$ & $\begin{array}{c}\text { Video } \\
\text { Ilama- } \\
\text { das }\end{array}$ & $\begin{array}{l}\text { Ed- } \\
\text { modo }\end{array}$ & $\begin{array}{l}\text { Flipped } \\
\text { Clas- } \\
\text { sroom }\end{array}$ & $\begin{array}{c}\text { Tareas } \\
\text { en } \\
\text { casa }\end{array}$ & $\begin{array}{l}\text { Video } \\
\text { You- } \\
\text { Tube }\end{array}$ & Zoom \\
\hline \multirow{4}{*}{$\begin{array}{l}\text { Whats- } \\
\text { App }\end{array}$} & $r$ & 1 & .168 & .085 & .271 & .078 & -.07 & .018 & .169 & .122 & -.05 \\
\hline & Sig & & .001 & .08 & .001 & .106 & .148 & .717 & .001 & .01 & .304 \\
\hline & $\mathrm{p}$ & & .40 & .29 & .52 & .27 & .26 & .13 & .41 & .34 & .22 \\
\hline & $1-\beta$ & & 1 & .99 & 1 & .99 & .99 & .99 & 1 & .99 & .99 \\
\hline \multirow[t]{4}{*}{ Facebook } & $r$ & .168 & 1 & .047 & .171 & .057 & -.014 & .004 & .064 & .004 & -.023 \\
\hline & Sig & .001 & & .331 & .001 & .237 & .768 & .339 & .191 & .34 & .637 \\
\hline & $\mathrm{p}$ & .40 & & .21 & .41 & .23 & .11 & .06 & .25 & .06 & .15 \\
\hline & $1-\beta$ & 1 & & .99 & 1 & .99 & .98 & .64 & .99 & .64 & .63 \\
\hline \multirow{4}{*}{$\begin{array}{l}\text { Platafor- } \\
\text { mas } \\
\text { institucio- } \\
\text { nales }\end{array}$} & $r$ & .085 & .047 & 1 & .157 & .051 & .057 & .05 & .081 & .087 & -.087 \\
\hline & Sig & .079 & .331 & & .001 & .299 & .242 & .299 & .095 & .074 & .079 \\
\hline & $p$ & .29 & .21 & & .39 & .22 & .23 & .22 & .28 & .29 & .29 \\
\hline & $1-\beta$ & .99 & .99 & & .99 & .99 & .99 & .99 & .99 & .99 & .99 \\
\hline \multirow{4}{*}{$\begin{array}{l}\text { Correo } \\
\text { electró- } \\
\text { nico }\end{array}$} & $r$ & .271 & .171 & .157 & 1 & .013 & .046 & -.005 & .183 & -.012 & -.034 \\
\hline & Sig & .001 & .001 & .001 & & .734 & .342 & 916 & .001 & .808 & .484 \\
\hline & $p$ & .52 & .41 & .39 & & .32 & .21 & .07 & .42 & .10 & .18 \\
\hline & $1-\beta$ & 1 & 1 & .99 & & 1 & .99 & .97 & 1 & .98 & .99 \\
\hline
\end{tabular}


Ulises Delgado Sánchez y Fernanda Gabriela Martínez Flores

\begin{tabular}{|c|c|c|c|c|c|c|c|c|c|c|c|}
\hline \multirow{4}{*}{$\begin{array}{l}\text { Video } \\
\text { Ilamadas }\end{array}$} & $r$ & .078 & .057 & .051 & .013 & 1 & -.093 & .034 & .205 & .178 & .124 \\
\hline & Sig & .106 & .237 & .293 & .794 & & .056 & .485 & .001 & .001 & .01 \\
\hline & $p$ & .27 & .23 & .22 & .11 & & .30 & .18 & .45 & .42 & .35 \\
\hline & $1-\beta$ & .99 & .99 & .99 & .98 & & .99 & .99 & 1 & 1 & .99 \\
\hline \multirow[t]{4}{*}{ Edmodo } & $r$ & -.07 & -.014 & .057 & .406 & -.093 & 1 & -.07 & -.085 & -.077 & -.017 \\
\hline & Sig & .148 & .768 & .242 & .342 & .056 & & .148 & .08 & .112 & .728 \\
\hline & $p$ & .26 & .26 & .23 & .63 & .30 & & .26 & .29 & .27 & .13 \\
\hline & $1-\beta$ & .99 & .99 & .99 & 1 & .99 & & .99 & .99 & .99 & .99 \\
\hline \multirow{4}{*}{$\begin{array}{l}\text { Flipped } \\
\text { Clas- } \\
\text { sroom }\end{array}$} & $r$ & .018 & .004 & .05 & -.005 & .034 & -.07 & 1 & .032 & .03 & -.089 \\
\hline & Sig & .717 & .939 & .30 & .91 & .485 & .148 & & .510 & .54 & .067 \\
\hline & $p$ & .13 & .06 & .22 & .07 & .18 & .26 & & .17 & .17 & .29 \\
\hline & $1-\beta$ & .99 & .97 & .99 & .96 & .99 & .99 & & .99 & .99 & .99 \\
\hline \multirow{4}{*}{$\begin{array}{l}\text { Tareas en } \\
\text { casa }\end{array}$} & $r$ & $.169 *$ & .064 & .081 & $.183 *$ & $.205^{*}$ & -.085 & .032 & 1 & $.164 *$ & .004 \\
\hline & Sig & .001 & .191 & .095 & .001 & .001 & .08 & .51 & & .001 & .93 \\
\hline & $p$ & .41 & .25 & .28 & .42 & .45 & .29 & .17 & & .40 & .06 \\
\hline & $1-\beta$ & 1 & .99 & .99 & 1 & 1 & .99 & .99 & & 1 & .97 \\
\hline \multirow{4}{*}{$\begin{array}{l}\text { Video } \\
\text { youtube }\end{array}$} & $r$ & $.122 *$ & .004 & .087 & -.012 & $.178 *$ & -.077 & .03 & $.164 *$ & 1 & $.147^{*}$ \\
\hline & Sig & .012 & .94 & .074 & .808 & .001 & .112 & .54 & .001 & & .002 \\
\hline & $p$ & .34 & .06 & .29 & .10 & .42 & .27 & .17 & .40 & & .38 \\
\hline & $1-\beta$ & .99 & .97 & .99 & .98 & 1 & .99 & .99 & 1 & & .99 \\
\hline \multirow[t]{4}{*}{ Zoom } & $r$ & -.050 & -.023 & -.087 & -.034 & $.124 *$ & -.017 & -.089 & .004 & $.147^{*}$ & 1 \\
\hline & Sig & .304 & .637 & .073 & .484 & .01 & .728 & .067 & .93 & .002 & \\
\hline & $p$ & .22 & .15 & .29 & .18 & .35 & .13 & .29 & .06 & $\cdot 38$ & \\
\hline & $1-\beta$ & .99 & .99 & .99 & .99 & .99 & .99 & .99 & .97 & .99 & \\
\hline
\end{tabular}

El empleo del WhatsApp estuvo correlacionado con el Facebook, el correo electrónico, tareas en clase y videos YouTube; mientras el Facebook correlacionó con el WhatsApp y el correo electrónico; las plataformas institucionales estuvieron correlacionadas con el correo electrónico; y a su vez, el correo correlacionó con el WhatsApp, Facebook, plataformas institucionales y las tareas en clase; la videollamada correlacionó con las tareas en casa, video en YouTube y Zoom; las tareas correlacionaron con el WhatsApp, el correo electrónico, videollamadas y videos en YouTube; por otra parte, los videos en YouTube correlacionaron con el WhatsApp, videollamadas, tareas en clase y Zoom; mientras que el Zoom correlacionó únicamente con las videollamadas y los videos en YouTube.

\section{Discusión}

En el presente estudio se logró identificar una actuación oportuna por parte de las instituciones de educación para la apertura y habilitación de plataformas institucionales como EVA, y así 
poder dar continuidad al proceso de enseñanza-aprendizaje en estos tiempos que la educación atraviesa por una crisis sanitaria de magnitud sin precedentes. Se encontró que $72.5 \%$ de los estudiantes han continuado sus clases por medio de plataformas virtuales institucionales, por lo que en la población estudiada fue el EVA implementado que presentó mayor empleo por parte de los docentes. A diferencia de los señalamientos de Del Prete, Cabrero y Halal (2018), estos resultados muestran la proactividad y el compromiso de las diferentes instituciones educativas en la implementación de la tecnología para favorecer la enseñanza y la aplicación de los EVA por medio de plataformas institucionales. Estas acciones impiden la emergencia de importantes barreras para el aprendizaje en momentos que la educación vive una crisis; lo cual permite vislumbrar que en este nuevo milenio y ante situaciones de crisis, las instituciones educativas están tomando medidas de contención para generar el menor impacto en la formación de los estudiantes.

A su vez, la implementación de plataformas institucionales ha sido de gran apoyo en la retroalimentación que los estudiantes reciben, al igual que el uso de la plataforma virtual institucional y el Zoom, lo que se evidencia por las correlaciones positivas en estas EVA y la retroalimentación hacia los estudiantes. Coincidentemente con Dallera y Tenaglía (2000), podemos considerar que el Zoom es un recurso útil para la enseñanza. Por ello, el uso de la plataforma institucional presentó una correlación positiva y significativa para proporcionar indicaciones por parte del profesor a los estudiantes, y continuar con la actividad de enseñanza.

La evidencia respalda que la implementación de un protocolo virtual de clases con la habilitación de plataformas virtuales institucionales como EVA contribuye a solventar y abatir el impacto educativo de la pandemia en la educación. Asimismo, la identificación del poco uso de recursos tecnológicos como Instagram, Microsoft Teams y Blogger, entre otros, parece implicar que, al generarse protocolos virtuales de aprendizaje, estas herramientas son las que presentan menor uso como EVA. Esto indica que aun cuando Microsoft Teams es una herramienta gratuita que puede soportar usos educativos (Microsoft, 2020), el diseño de las plataformas institucionales ha permitido homogeneizar en gran medida la aplicación de este EVA en tiempos de crisis, favoreciendo que los docentes hagan uso de ellas para lograr un mayor seguimiento institucional de la formación educativa de los jóvenes en diferentes niveles.

Los docentes no solo se valieron de un EVA en exclusiva para poder continuar con la enseñanza, sino que se apoyaron con diversos recursos para tal objetivo, siendo el WhatsApp, el segundo EVA más usado por los profesores con $58.1 \%$ de uso, lo cual nos permite coincidir con Suárez (2018) y Trejo (2018), quienes le ubican como un mecanismo rápido para dar anunciación; pero a diferencia de estos autores, la presente investigación no encontró una correlación significativa entre el WhatsApp y la retroalimentación, lo cual permite afirmar que si bien es un EVA de rápido acceso al estar en los celulares como una aplicación de uso diario, esta no parece considerarse eficiente al momento de realizar una retroalimentación para los estudiantes. 
Por otra parte, el correo electrónico es una herramienta que usan casi todas las personas que tienen acceso a internet y algún dispositivo inteligente (Puerta, Sánchez, 2010). Este EVA parece ser de mayor utilidad al momento de dar indicaciones, pero no para una retroalimentación por parte del docente, por lo que se considera un EVA parcialmente eficiente en el proceso de enseñanza-aprendizaje, aunque solo para dar indicaciones.

Es importante destacar, como ya se mencionó, que los docentes utilizaron varios EVA de manera complementaria, lo cual hace patente que las competencias tecnológicas en los docentes son adecuadas para implementar estrategias de enseñanza aprendizaje con los estudiantes en diversos EVA. Se encontró que, a mayor uso de videollamadas, tienden a usar también videos educativos en YouTube, tareas en casa y Zoom, así como una asociación notable entre el uso de plataformas institucionales y correo electrónico.

Si bien es cierto que en esta investigación se encontró que las plataformas institucionales, el WhatsApp y el correo son los EVA de mayor uso en la presente contingencia, se identificó que el uso de las plataformas, ya sea las institucionales o Zoom, permitieron la retroalimentación oportuna de los estudiantes. Esto deja ver que cada vez el uso de los EVA ha ido permeando a las instituciones de educación, y son una excelente herramienta de apoyo en momentos que la educación está en crisis, no solo de pandemia sino también en algunas otras situaciones que pudieran derivarse de otros desastres naturales o sociales. Futuras investigaciones podrían dirigirse a identificar los equipos y dispositivos por medio de las cuales los estudiantes y docentes usan los EVA, ya sean celulares, computadoras portátiles, tabletas y computadoras fijas, y la accesibilidad a internet, para que se puedan ir desarrollando en la educación durante épocas de crisis, así como el conocimiento de espacios que favorezcan su aprendizaje.

Esto deja una ventana de oportunidad a las instituciones de educación para realizar una migración de sus contenidos a plataformas virtuales, si bien no se puede virtualizar todos los contenidos y prácticas educativas, sí se pueden generar plataformas de apoyo permanente a la educación, a fin de fortalecer y eventualmente desplazar a la educación tradicional. En futuras investigaciones se tendrán que identificar los EVA que se implementaron en educación básica y los efectos en el aprendizaje de los estudiantes.

\section{Referencias}

Abúndez, E.; F. Fernández; L. Meza; M. Álamo (2015). Facebook como herramienta educativa en el proceso de enseñanza-aprendizaje en el nivel medio superior. Zona Próxima, (22), 116127. https://www.redalyc.org/articulo.oa?id=85339658009

Aguilera, C.; A. Manzano; I. Martínez; M. C. Lozano; C. Casiano (2017). El modelo Flipped Classroom. International Journal of Developmental and Educational Psychology, 4(1), 261-266. http://doi.org/10.17060/ijodaep.2017.n1.v4.1055

Diálo pos sobre Educación año 12 | número 22 | enero-junio 2021 | ISSN 2007-2171 
Álvarez, H.; E. Arias; A. Bergamaschi; A. López; A. Noli; A. Viteri (2020). La educación en tiempos del coronavirus. Los sistemas educativos de América Latina y el Caribe ante COVID-19. Banco Interamericano de Desarrollo. https://bit.ly/2HiHbla

Aranciba, M. L.; J. Cabrero; I. Valdivia (2019). Comparative study between teachers and students on acceptance and use of technologies for educational purposes in the Chilean context. Apertura, 11(1), 1-20.http://dx.doi.org/10.32870/Ap.v11n1.1440

Ardila, M. (2011). Indicadores de calidad de las plataformas educativas digitales. Educación y Educadores, 14(1), 189-206.

Blanco, A.; R. López (2017). La posibilidad pedagógica de la red social Instagram para aproximar a los estudiantes al género poético. En Ruiz, J.; J. Sánchez; E. Sánchez (eds.). Innovación docente y uso de las TIC en educación. Málaga: UMA Editorial.

Dallera, M.; P. Tenaglia (2000). La videoconferencia como resultado didáctico TIC. Problematización y debate desde las ciencias sociales. Congreso Iberoamericano de Ciencia, Tecnología, Innovación y Educación, 1-18.

Del Prete, A.; J. Cabrero (2019). Las plataformas de formación virtual: algunas variables que determinan su utilización. Apertura, 11(2), 138-153. http://dx.doi.org/10.32870/Ap.v11n2.1521

Del Prete, A.; J. Cabrero; C. Halal (2018). Motivos inhibidores del uso de Moodle en docentes de educación superior. Campus virtuales. Revista Científica Iberoamericana de Tecnología Educativa, 7(2), 69-80 http://www.uajournals.com/ojs/index.php/campusvirtuales/article/ view/347/271

Díaz, S. (2009). Plataformas educativas, un entorno para profesores y alumnos. Revista Digital para Profesionales de la Enseñanza, (2), 1-7

Ferreriro, V. V.; A. A. Garambullo; J. Brito (2013). Prácticas innovadoras: uso de la plataforma black-board en modalidades semipresenciales. Caso práctico UABC FIN Tecate. Revista lberoamericana para la Investigación y Desarrollo Educativo, 4(7), 129-150.

Fietyat, Y.; L. Ahmad; P. Yudi (2017). A Proposed Model for Investigating on Web WhatsApp Application. Advanced Science Letters, 23(5), 4050-4054. https://doi.org/10.1166/asl.2017.8308 Lorenzo, B. (2019). Google Classroom como herramienta didáctica para trabajar las destrezas de compresión lectora y de expresión escrita en inglés. Tesis de Maestría. España: Universidad de la Laguna. https://bit.ly/391yddT

Luis, E. (2014). El uso del correo electrónico como recurso didáctico: el caso del centro Institut Escolar del Treball de Barcelona. Tesis de Maestría. Barcelona: Universidad Internacional de La Rioja.

Lloret, S.; A. Ferreres; A. Hernández; I. Tomás (2014). Análisis factorial exploratorio de los items: una guía práctica, revisada y actualizada. Anales de Psicología, 20(3), 1151-1169. http://scielo.isciii.es/scielo.php?script=sci arttext\&pid=S0212-97282014000300040

Mejía, P. G.; K. P. Hidalgo; A. A. Rosero; W. E. Yugsan (2018). La plataforma Edmodo como recurso didáctico en la enseñanza de la escritura académica del idioma inglés. INNOVA Research Journal, 3(6), 97-107. http://revistas.uide.edu.ec/index.php/innova/article/view/558 
Microsoft (2020). El aprendizaje remoto, colaborativo y seguro es gratis con Microsoft Teams. https://bit.ly/3|KnOBS

Organización de las Naciones Unidas para la Educación, la Ciencia y la Cultura (2020). La educación en situaciones de crisis. https://es.unesco.org/themes/educacion-situaciones-crisis

Organización de las Naciones Unidas (2020). Información de políticas: la educación durante la COVID-19 y después de ella. https://www.un.org/sites/un2.un.org/files/policy brief - education during covid-19 and beyond spanish.pdf

Pérez. A.; A. Cuecuecha (2019). El efecto de usar YouTube como apoyo didáctico en calificaciones de microeconomía. Apertura, 11(2), 22-39. http://dx.doi.org/10.32870/Ap.v11n2.1650

Puerta. C. A.; A. Sánchez (2010). El correo electrónico: herramienta que favorece la interacción en ambientes educativos virtuales. Revista Virtual Universitaria Católica del Norte, (30), 1-27.

Quirós, E. (2009). Recursos didácticos digitales: medios innovadores para el trabajo colaborativo en línea. Revista Electrónica Educare, 13(2), 47-62.

Ricardo, C.; E. Manotas; B. Ballesteros; A. Peláez; V. Vila; S. Zapata; A. Chavarro (2017). Uso del video educativo en la educación superior. En Ricardo, C.; F. Iriarte (eds.). Las TIC en la educación superior, experiencia de innovación. Bogotá: Universidad del Norte, 85-122.

Ruíz, M.; V. Abella (2011). Creación de un blog como herramienta TIC e instrumento TAC en el ámbito universitario. Teoría de la Educación. Educación y Cultura en la Sociedad de la Información, 12(4), 53-70. http://www.redalyc.org/articulo.oa?id=201022649005

Rodríguez M. C.; J. Fernández (2017). Uso del recurso de contenido en el aprendizaje en línea: YouTube. Apertura, 9(1), 22-31. http://dx.doi.org/10.32870/Ap.v9n1.1018

Suárez, B. (2018). WhatsApp: su uso educativo, ventajas y desventajas. Revista de Investigación en Educación, 16(2), 121-135. http://webs.uvigo.es/reimed/

Trejo, O. I. (2018). WhatsApp como herramienta de apoyo al proceso de enseñanza y aprendizaje de la programación de computadores. Educación y Ciudad, (35), 149-158. http://dim.pangea.org/revistaDIM30/docs/OC30whatsapp.pdf 